\title{
Succinylcholine-Induced Postoperative Myalgia: Etiology and Prevention
}

\author{
Shabana Zainab Shafy ${ }^{a}$, , Mohammed Hakima ${ }^{\text {a }}$, Senthil G. Krishna, ${ }^{a}$, \\ Joseph D. Tobias ${ }^{\mathrm{a}, \mathrm{b}, \mathrm{c}}$
}

\begin{abstract}
Succinylcholine is a neuromuscular blocking agent frequently used to facilitate endotracheal intubation during general anesthesia. It is usually preferred due to its rapid onset of action and short duration of action. However, the effectiveness of this drug is limited by certain adverse effects, one of which is postoperative myalgia. Although generally considered a minor adverse effect, the myalgia may be severe and interfere with return to normal activities of daily life. We present an 18-year-old with a history of ulcerative colitis who presented for anesthetic care during liver biopsy. Due to comorbidities including obesity, succinylcholine was chosen to provide rapid neuromuscular blockade and facilitate endotracheal intubation. Postoperatively, the patient complained of bilateral calf pain. The etiology of muscle pain related to succinylcholine is discussed and potential strategies to reduce its incidence reviewed.
\end{abstract}

Keywords: Succinylcholine; Myalgia; Anesthesia

\section{Introduction}

Succinylcholine is a rapidly acting, depolarizing neuromuscular blocking agent (NMBA) that is used to facilitate endotracheal intubation. It acts by mimicking the effect of acetylcholine at the neuromuscular junction thereby producing depolarization of the sarcolemma. As its degradation is dependent on pseudocholinesterase and not acetylcholinesterase, it continues to occupy the receptor thereby providing neuromuscular blockade for up to $10 \mathrm{~min}$. Although its onset

Manuscript submitted June 4, 2018, accepted June 27, 2018

aDepartment of Anesthesiology and Pain Medicine, Nationwide Children's Hospital, Columbus, OH, USA

${ }^{b}$ Department of Anesthesiology and Pain Medicine, The Ohio State University College of Medicine, Columbus, OH, USA

'Department of Pediatrics, The Ohio State University College of Medicine, Columbus, OH, USA

${ }^{\mathrm{d} C o r r e s p o n d i n g ~ A u t h o r: ~ S h a b a n a ~ Z a i n a b ~ S h a f y, ~ D e p a r t m e n t ~ o f ~ A n e s t h e s i o l o-~}$ gy and Pain Medicine, Nationwide Children's Hospital, 700 Children's Drive, Columbus, OH 43205, USA. Email: shabana.shafy@nationwidechildrens.org

doi: https://doi.org/10.14740/jmc3107w is rapid making it useful when emergent securement of the airway is needed, the usefulness of the drug is restricted by rare yet significant adverse effects [1,2]. Life-threatening adverse effects such as hyperkalemia are more common in the presence of specific comorbid conditions thereby contraindicating its use in such clinical scenarios [2,3]. Given these concerns, its use is generally restricted to clinical scenarios where there are concerns of a potentially difficult airway or when rapid securement of the airway is indicated.

Given its mechanism of action with depolarization of the sarcolemma, muscle pain of variable intensity and duration may occur $[4,5]$. Bourne et al were the first to report postoperative myalgia in 1952 as well as the first to attempt to delineate its mechanism and reduce its incidence and severity by pretreatment with gallamine [6,7]. Although several regimens or pretreatments have been tried in an effort to decrease the incidence or severity of myalgias with succinylcholine, the effectiveness of such measures is debatable [8]. We present an 18-year-old patient with ulcerative colitis who presented for anesthetic care during liver biopsy. Due to concerns of obesity and delayed gastric emptying, succinylcholine was chosen to provide rapid neuromuscular blockade and facilitate endotracheal intubation. Postoperatively, the patient complained of bilateral calf pain. The etiology of muscle pain related to succinylcholine is discussed and potential strategies to reduce its incidence reviewed.

\section{Case Report}

Institutional Review Board approval is not required for presentation of single case report at Nationwide Children's Hospital (Columbus, OH). The patient was an 18-year-old, 129.8 $\mathrm{kg}$ adolescent with a body mass index (BMI) of $41 \mathrm{~kg} / \mathrm{m}^{2}$ who was scheduled for a liver biopsy for suspected non-alcoholic fatty liver disease (NAFLD). His past history was positive for ulcerative colitis which had previously required laparoscopic colectomy with colostomy. Other past surgeries included adenotonsillectomy and ventral hernia repair. There was no history of previous problems with general anesthesia during any of the procedures. Current medications included cholecalciferol ( 1,000 unit tablet) once daily and metronidazole $0.75 \%$ topical gel. The patient had known allergies to penicillin and cephalosporins. Due to obstructive sleep apnea, he used patient Bilevel Positive Airway Pressure (BiPAP) at night. On the morning of 
the procedure, his physical examination and preoperative vital signs were unremarkable. The anesthetic plan, risks, benefits and alternatives to general anesthesia were discussed with the parent and informed consent obtained. The patient was held nil per os for $8 \mathrm{~h}$ and was transported to the operating room. Routine American Society of Anesthesiologists' monitors were placed. The patient was allowed to breathe $70 \%$ nitrous oxide in oxygen followed by placement of a peripheral intravenous cannula. Anesthesia was induced with propofol $(200 \mathrm{mg})$ and neuromuscular blockade facilitated by the administration of succinylcholine $(140 \mathrm{mg})$. Maintenance anesthesia included sevoflurane $(2-3 \%)$ in air/oxygen with fentanyl $(100 \mu \mathrm{g})$. Additional medications included dexamethasone $(8 \mathrm{mg})$ and ondansetron (4 mg). The procedure lasted approximately $35 \mathrm{~min}$. At the completion of the procedure, the patient's trachea was extubated and he was transported to the post-anesthesia care unit. In the immediate postoperative period, he complained of pain in both his lower extremities, mainly in the calf muscles, for which he was offered various treatment options, which he chose to defer due to concerns related to the use of additional opioids. Over the course of the next $1-2 \mathrm{~h}$, the calf pain slowly resolved and he was discharged home. The remainder of his postoperative course was unremarkable.

\section{Discussion}

Succinylcholine is a NMBA with a very rapid clinical onset of action, making it an ideal agent for neuromuscular blockade when rapid securing of the airway is indicated. Despite its significant advantages, its use has been limited by concerns regarding its adverse effect profile including severe myalgias. The incidence of postoperative myalgia due to succinylcholine varies, being as high as $90 \%$ and generally thought to be approximately $50 \%[4,9]$. The myalgias have been described as similar to the pain or muscle soreness experienced after a significant physical activity [10]. The duration and intensity of this discomfort is highly variable.

The mechanism for the postoperative myalgia has not completely been elucidated. Postulated mechanisms include increased intracellular calcium concentrations, membrane phospholipid degradation, and release of free fatty acids and free radicals, which lead to increased membrane permeability [11-13]. It has been proposed that the pain occurs due to muscle damage produced by shearing forces associated with the fasciculations at the onset of phase one block [14]. Studies demonstrating electromyographic spike trains of succinylcholine-induced fasciculations in patients have shown the possible development of microdamage at the extrafusal muscles [15]. While there is no correlation between the elevation of creatine phosphokinase and the development of muscle pain, the increase in the serum potassium concentration is greater in patients who develop myalgia than in those who do not $[16,17]$.

Although bothersome, myalgias following the administration of succinylcholine are generally self-limited although they may interfere with a rapid return to activities of daily life including return to work or school [18]. Various demograph- ic risk factors have been identified. Myalgias occur less frequently in children and patients over 50 - 60 years of age, less commonly in females, and less commonly during pregnancy possibly due to hormonal influences $[9,19,20]$. The state of training of the muscle has also been implicated, with a lower incidence and decreased severity of pain in patients with better muscular fitness [10]. Patients who undergo minor procedures and those who are ambulated earlier are at higher risk for the development of postoperative myalgias [7, 21].

Various methods and regimens have been evaluated to decrease the incidence and severity of the myalgias. Pre-treatment with a small dose of a non-depolarizing neuromuscular blocking agent prior to the administration of succinylcholine, remains the most commonly employed method, dating back to a 1957 report of the use of gallamine [7]. Other methods including stretching exercises and supplementation of vitamin $\mathrm{C}$, have been reported to be effective $[22,23]$. Since it has been postulated that calcium influx enhances the intensity of muscle fiber contractions, inducing spindle damage and subsequent pain, pre-treatment with medications such as dantrolene which interfere with intracellular calcium release have been reported to be effective [24]. It has also been demonstrated that pre-treatment with calcium gluconate and lidocaine, which provide membrane stabilizing actions, are protective $[25,26]$. Comparative studies between the inductions of anesthesia with propofol versus thiopental have shown a decreased association of myalgias after induction with propofol [27].

In summary, a high incidence of postoperative myalgia has been documented following the administration of succinylcholine. While generally self-limited, the myalgias can result in significant pain, resulting in a delay of return to work or school. As outlined above, demographic and clinical factors may increase the incidence and severity of the pain. Various techniques have been described to control the problem, the most commonly used technique being the administration of a small dose of a non-depolarizing neuromuscular blocking agent 2 - 3 min before the administration of succinylcholine. While effective, this technique may delay the onset of succinylcholine and also increase the dose requirements. As such, it is not recommended in emergency situations when succinylcholine is used to rapidly secure the airway. Most importantly, controversy remains regarding the administration of succinylcholine. While its routine use is not recommended due to concerns of hyperkalemia in patients with undiagnosed myopathy, the manufacturer's package insert still describes its use for emergency tracheal intubation or in instances where immediate securing of the airway is necessary [28-30].

\section{Funding}

None.

\section{Conflict of Interest}

None. 


\section{Author Contributions}

All authors contributed equally to this manuscript.

\section{References}

1. Tobias JD. Rapid sequence intubation: What does it mean? Does it really matter? Saudi J Anaesth. 2014;8(2):153-154.

2. Martyn JA, Richtsfeld M. Succinylcholine-induced hyperkalemia in acquired pathologic states: etiologic factors and molecular mechanisms. Anesthesiology. 2006;104(1):158-169.

3. Gronert GA. Succinylcholine-induced hyperkalemia and beyond. 1975. Anesthesiology. 2009;111(6):1372-1377.

4. Wong SF, Chung F. Succinylcholine-associated postoperative myalgia. Anaesthesia. 2000;55(2):144-152.

5. Pace NL. Prevention of succinylcholine myalgias: a meta-analysis. Anesth Analg. 1990;70(5):477-483.

6. Bourne JG, Collier HO, Somers GF. Succinylcholine (succinoylcholine), muscle-relaxant of short action. Lancet. 1952;1(6721):1225-1229.

7. Churchill-Davidson HC. Suxamethonium (succinylcholine) chloride and muscle pains. $\mathrm{Br}$ Med J. 1954;1(4853):74-75.

8. Mencke T, Schreiber JU, Becker C, Bolte M, Fuchs-Buder T. Pretreatment before succinylcholine for outpatient anesthesia? Anesth Analg. 2002;94(3):573-576; table of contents.

9. Crawford JS. Suxamethonium muscle pains and pregnancy. Br J Anaesth. 1971;43(7):677-680.

10. Newnam PT, Loudon JM. Muscle pain following administration of suxamethonium: the aetiological role of muscular fitness. Br J Anaesth. 1966;38(7):533-540.

11. Suarez-Kurtz G, Eastwood AB. Release of sarcoplasmic enzymes from frog skeletal muscle. Am J Physiol. 1981;241(3):C98-105.

12. McLoughlin C, Nesbitt GA, Howe JP. Suxamethonium induced myalgia and the effect of pre-operative administration of oral aspirin. A comparison with a standard treatment and an untreated group. Anaesthesia. 1988;43(7):565-567.

13. Mizogami M, Fujibayashi T, Goto Y. Succinylcholineinduced fasciculations in denervated rat muscles as measured using 31P-NMR spectroscopy: the effect of pretreatment with dantrolene or vecuronium. Acta Anaesthesiol Scand. 1998;42(4):472-477.
14. Waters DJ, Mapleson WW. Suxamethonium pains: hypothesis and observation. Anaesthesia. 1971;26(2):127141.

15. Poon PW, Lui PW, Chow LH, Lam FK, Chan FH, Lin YM. EMG spike trains of succinylcholine-induced fasciculations in myalgic patients. Electroencephalogr Clin Neurophysiol. 1996;101(3):206-210.

16. Tammisto T, Airaksinen MM. Suxamethonium-induced myoglobinuria. Br J Anaesth. 1965;37:464-465.

17. Collier CB. Suxamethonium pains and early electrolyte changes. Anaesthesia. 1978;33(5):454-461.

18. van den Berg AA, Iqbal S. Post suxamethonium myalgia - will we never learn? Anaesth Intensive Care. 1996;24(1):116-117.

19. Foster CA. Muscle pains that follow administration of suxamethonium. Br Med J. 1960;2(5191):24-25.

20. Riding JE. Minor complications of general anaesthesia. Br J Anaesth. 1975;47(2):91-101.

21. Brodsky JB, Ehrenwerth J. Postoperative muscle pains and suxamethonium. Br J Anaesth. 1980;52(2):215-218.

22. Magee DA, Robinson RJ. Effect of stretch exercises on suxamethonium induced fasciculations and myalgia. Br J Anaesth. 1987;59(5):596-601.

23. Gupte SR, Savant NS. Post suxamethonium pains and vitamin C. Anaesthesia. 1971;26(4):436-440.

24. Laurence AS. Oral dantrolene prevents rise of myoglobin due to suxamethonium. Anaesthesia. 1985;40(9):907910.

25. Shrivastava OP, Chatterji S, Kachhawa S, Daga SR. Calcium gluconate pretreatment for prevention of succinylcholine-induced myalgia. Anesth Analg. 1983;62(1):5962.

26. Chatterji S, Thind SS, Daga SR. Lignocaine pretreatment for suxamethonium. A clinicobiochemical study. Anaesthesia. 1983;38(9):867-870.

27. McClymont C. A comparison of the effect of propofol or thiopentone on the incidence and severity of suxamethonium-induced myalgia. Anaesth Intensive Care. 1994;22(2):147-149.

28. Rosenberg H, Gronert GA. Intractable cardiac arrest in children given succinylcholine. Anesthesiology. 1992;77(5):1054.

29. Bingham R, Battersby E, Black A, Facer E, Hatch D, Howard R, James I, et al. Succinylcholine warning. Can J Anaesth. 1994;41(7):657-658.

30. Morell RC. FDA group urges sux label reduced to "warning". J Clin Monit. 1995;11(2):141-143. 